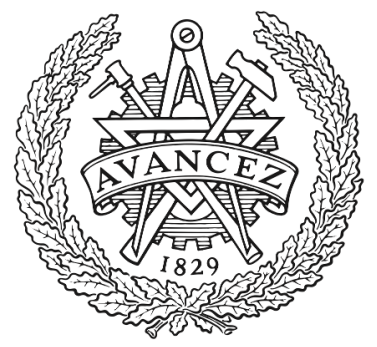

CHALMERS

UNIVERSITY OF TECHNOLOGY

\title{
Preparing for GBit/s coverage in 5G: Massive MIMO, PMC packaging by gap waveguides, OTA testing in random-LOS
}

Downloaded from: https://research.chalmers.se, 2023-04-26 08:54 UTC

Citation for the original published paper (version of record):

Kildal, P. (2015). Preparing for GBit/s coverage in 5G: Massive MIMO, PMC packaging by gap waveguides, OTA testing in random-LOS. Loughborough Antennas and Propagation Conference, LAPC 2015, Burleigh Court International Conference Centre, Loughborough University, United Kingdom, 2-3 November. http://dx.doi.org/10.1109/LAPC.2015.7365987

N.B. When citing this work, cite the original published paper. 


\title{
Preparing for GBit/s Coverage in 5G:
}

\author{
Massive MIMO, PMC Packaging by Gap Waveguides, OTA Testing in Random-LOS
}

\author{
Per-Simon Kildal \\ Division of Antenna Systems, Department of Signal and Systems \\ Chalmers University of Technology \\ 41296 Gothenburg, Sweden \\ per-simon.kildal@chalmers.se
}

\begin{abstract}
In this invited presentation I describe how we prepare for $5 \mathrm{G}$ in $\mathrm{my}$ research division. The background is that we have contributed to $3 G$ and $4 G$ developments with two commercial successes: the hat-fed reflector antenna for backhaul radio links, and Bluetest reverberation chambers for OTA (OerThe-Air) characterization of devices with MIMO and OFDM.

5G means Gbit/s data rates, for which higher frequencies are needed towards the user terminal, may be up to 30 or $60 \mathrm{GHz}$. This means that the multipath will be weaker as it gradually diminishes when frequency increases. Therefore, we prepare by introducing a Random-LOS (RLOS) complement to the OTA testing in Rich Isotropic Multipath (RIMP) being provided by Bluetest's reverberation chambers.

Further, high gain steerable beams will be needed, requiring new planar packaging solutions for closer integration of antennas and RF chipsets. Therefore, we prepare by research on gap waveguides. Massive MIMO have many technological uncertainties, so others also consider old-fashioned phased arrays, but they will not work in RIMP.

The best hardware can only be chosen if we know how to characterize the system performance. The radiation pattern and realized gain cannot be used directly for this purpose due to all the statistical variations caused by the arbitrariness of the user. Therefore, we introduce instead the Probability of Detection (PoD) as a quality metric. This will be different for each desired number of bitstreams. Further, we quantify the difference between different PoD curves in dBiid in RIMP, i.e. in $\mathrm{dB}$ relative to the i.i.d. (idependent identically distributed) case, and in $\mathrm{dBt}$ in RLOS, i.e. in $\mathrm{dB}$ relative to an ideal polarization- and coveragematched threshold receiver.
\end{abstract}

The presentation will give an overview of this research.

Keywords-OTA testing; Massive MIMO; EM packaging; gap waveguides; RIMP; Random-LOS; threshold receiver

\section{INTRODUCTION}

The wireless evolution from $1 \mathrm{G}$ (e.g. NMT) systems in 1995 towards 4G LTE launched in 2009 has gone very fast. 4G also grows fast due to the wide-spread use of smart-phones, with which we have started to approach the paperless society. The wireless industry and research environments are now competing in guessing how the next 5 th wireless generation (5G) will look like and when it will come. Most people associate $5 \mathrm{G}$ with $\mathrm{Gbit} / \mathrm{s}$ data rate to the user terminal, and expect it to appear commercially around year 2020. There are already many key-words describing how the 5G antenna system will look like, and the two most frequently used ones are Massive MIMO and millimeter waves. MIMO (and OFDM) is in $4 \mathrm{G}$ used to combat the negative effects of the fading, and also to exploit the potential of the multipath propagation. In 5G we need Massive MIMO in order to get sufficient gain and steer-ability at millimeter wave frequencies (Figure 1). The millimeter waves are needed to get sufficient bandwidth for the GBit/s data rates.

However, when the frequencies increase up to $30 \mathrm{GHz}$ and may be even $60 \mathrm{GHz}$, the multipath propagation and fast fading gradually vanish. As a consequence, the classical Line-OfSight (LOS) contribution becomes dominant. This causes another type of statistical signal variation to be prevalent in $5 \mathrm{G}$ wireless systems, corresponding to what in the literature is referred to as slow fading. We have introduced the term Random-LOS to describe this randomness, and some fundamental metrics to characterize it, to be explained in Section III. Measurements at $60 \mathrm{GHz}$ have shown that there could also be a single dominant wave contribution if the user is shadowed by buildings, such as waves diffracted around corners of buildings [1]. Therefore, we include also such single dominant wave contribution in the Random-LOS concept. Thus, the LOS term shall not be interpreted literally in the Random-LOS expression.

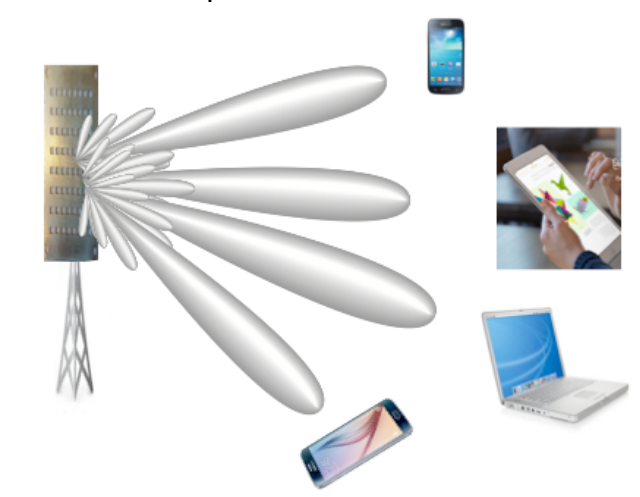

Fig. 1. Simplified illustration of a 5G Massive MIMO system with a base station providing 4 reconfigurable beams to each of 4 users which have LOS but with random AoA and polarization (due to random orientation of terminal).

The purpose of the present paper is to give an overview how we at Chalmers prepare for contributing to $5 \mathrm{G}$ by developing i) a conceptually new waveguide technology for 
planar array antenna systems with integrated RF front-ends at $60 \mathrm{GHz}$, and ii) an OTA test concept for Random-LOS environments. The new waveguide concept is termed gap waveguides, and it is built on previous works on soft and hard surfaces. The soft and hard surfaces represent an early metasurface concept built around corrugated surfaces, and the gap waveguides is based on realizing a Perfect Magnetic Conductor (PMC) by using metal pins or other periodic surfaces. The soft surface has resulted in a successfully commercialized reflector antenna for radio links. On the other hand, the Random-LOS OTA concept is based on the knowhow built up during the last 15 years for characterization of $3 \mathrm{G}$ and $4 \mathrm{G}$ wireless terminals and devices in reverberation chambers, being successfully commercialized by Bluetest AB.

Our two $5 \mathrm{G}$ concepts and their $3 \mathrm{G}-4 \mathrm{G}$ backgrounds are described in Sections II and III, respectively.

\section{DiRECTIVE ANTENNA SySTEMS DEVELOPED FROM METASURFACE CONCEPTS}

\section{A. Comhat reflector antennas for fixed beam in $3 G$ Backhaul}

The backhaul network in telecommunications is built both by optical fibers and radio links. The radio links are more popular because they are not dependent on being dug into the ground. Radio links exist for specific frequency bands between 6 and $42 \mathrm{GHz}$, and recently also $\mathrm{V}$-band (around $60 \mathrm{GHz}$ ) and E-band 70-90 GHz. The far most popular antenna solutions are reflector antennas with diameters ranging from $30 \mathrm{~cm}$ to $1.8 \mathrm{~m}$.

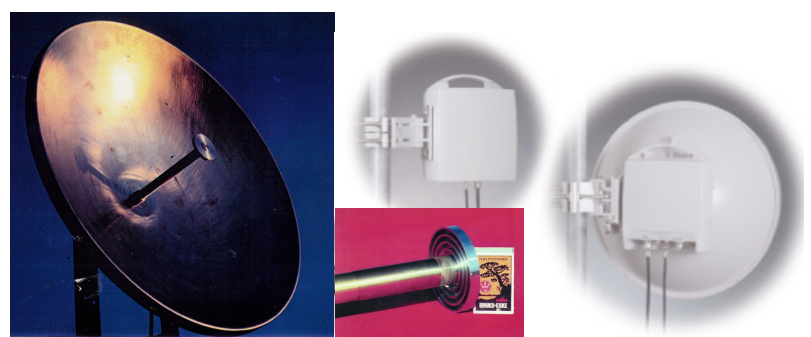

Fig. 2. The original hat feed in ring-focus reflector originally developed for satellite-TV reception at $12 \mathrm{GHz}$ (left). The inserted small picture shows the feed with a matchbox behind it. The innovative use of a corrugated soft-type metasurface is clearly seen. The photos in the background to the right are hatfed reflectors as used in Ericsson's MINI-LINK for two different reflector sizes.

The hat feed was first published in 1987. This invention was a result of several years experience with designing primefocus reflectors and feeds, and in particular corrugated horn feeds, as described in [2]. The corrugated horn work resulted in a generalization of corrugated surfaces to the concept of soft and hard surfaces [3]. The first application of this concept was to design the hat feed, in which the corrugations were used to make the far-field function rotationally symmetric with low cross polarization. Thereby it became possible to design prime-focus reflector antennas with very low sidelobes that were much more cost-effective and compact than previous symmetrical reflectors used in radio links. I had several attempts to commercialize it, but did not succeed before the $3 \mathrm{G}$ system required the backhaul to be built out with more affordable radio links. Then, we had also got better computational control of the bandwidth. Since year 2000 the hat-fed reflector (also referred to as the Comhat antenna) has been produced in more than one million copies mainly for Ericsson's MINI-LINK product (Figure 2). The production was in Sweden until 2013. Then it was moved to Hungary in order to reduce cost, and the volumes started to increase again.

\section{B. Gap waveguides for $60 \mathrm{GHz}$ Massive MIMO antenna systems in $5 G$}

The $5 \mathrm{G}$ system will require affordable reconfigurable directive antennas in the base stations to provide personalized services to the users (Figure 1). The higher millimeter wave frequencies do not propagate through walls and vegetation. Therefore, there must also be many more base stations than before. There is also larger atmospheric attenuation than at lower frequencies. This cannot be compensated by using larger antennas, because then the beam widths become too narrow and there will be problems with the required pointing accuracy.

We believe that new waveguide technologies are needed to build up the RF parts of Massive MIMO base stations. The reason is that the transceivers and the antenna must be integrated in one single unit without mechanical interfaces. The gap waveguide is such a new technology. The gap waveguide makes use of the modal cutoff in the narrow gap between a metal surface and a PMC, in order to control wave propagation along metal ridges or grooves in the PMC, or dielectricsupported metal strips in the gap. The cutoff is present within a large stopband that easily has decade bandwidth.

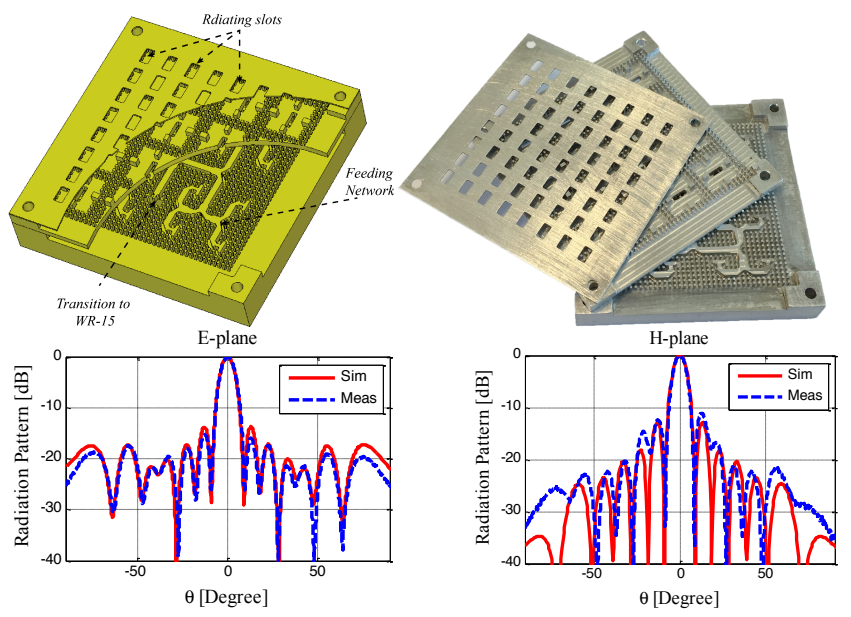

Fig. 3. $8 \times 8$ slot array waveguide realized by three metal layers and its radiation patterns in the middle of the $60 \mathrm{GHz}$ band. The middle layer consists if $4 \times 4$ groove gap waveguide cavities, and the lower forms a ridge gap waveguide distribution network. The antenna has an aperture efficiency including losses in the distribution network above $80 \%$ at 57 to $64 \mathrm{GHz}$.

The work on artificial soft and hard surfaces was stimulated by others research on EBG surfaces after 2000. We tried to conceptually distinguish the different surfaces in a simple way by introducing a table of canonical surfaces in 2005 [4]. This made it simpler to think further.

The gap waveguides is a further development of the corrugated hard-type surface, i.e. the canonical longitudinal PEC/PMC strip grid. We discovered local waves along each individual ridge between the corrugations when a parallel metal surface was located above them. The geometry was then refined to support a single local wave in different ways, i.e. a gap waveguide [5]. The gap waveguides have already 
demonstrated that it can be used to make high-Q filters, which is needed for separating receiving and transmitting frequency bands [6]. It can also be used as a general PMC packaging technology for integrating active and passive millimeter wave components [7]. Recently we have also demonstrated directive gap waveguide antennas with a realized gain above $24 \mathrm{dBi}$ [8]. The latter is shown in Figure 3.

We are now working to develop this technology further, and we think the gap waveguides will be an attractive solution also for Massive MIMO arrays, but then there will be filters and MMICs integrated within the distribution network. Then, it will be possible to control each subarray to achieve the required scan capability of the full array within limits. The details of Massive MIMO array architectures are not yet known, and some people envision them more as normal phased arrays with phase-only control of each element or subarray. Then, the requirements to signal processing resources may be reduced, but the flexible multi-user approach is lost.

\section{RIMP AND RANDOM-LOS OTA TEST CONCEPTS}

\section{A. RIMP concept commercialized for $3 G$ and $4 G$ by Bluetest}

I started Bluetest AB in year 2000. The idea was to develop an alternative to anechoic chambers for testing of mobile phones, based on a conviction that radiation pattern shapes did not really matter in multipath. We showed early that the reverberation chamber could provide a rich isotropic multipath environment, if properly designed. Later we introduced the abbreviation RIMP as the generic name. We showed that antenna diversity and MIMO performance could be determined for multi-port arrays in reverberation chambers, and we defined different diversity gains [9] -[10] that are well accepted today. The big opportunity for Bluetest came with the introduction of the 4G LTE system in 2009. Then, Bluetest had already developed a procedure for measuring the data rate throughput of LTE devices, and sales started to increase. The real breakthrough came a few years later, and Bluetest has till now sold more than 200 test chambers worldwide. [11] summarizes the reverberation chamber test concept until 2012.

The past years' developments have progressed fast after we found that digital receivers work as threshold receivers [12]. The modulation and coding makes the transition very steep between not detecting and detecting the bitstream. The detection appears within a few tenth of $\mathrm{dB}$ increase of the transmitted power for the AWGN case, i.e. the stationary case without fading. This insight made it possible to develop simple models for predicting the throughput in LTE and WiFi systems enabled for MIMO and OFDM [13]. The simple models make use of the basic MRC (Maximal Ratio Combining) and ZF (Zero Forcing) algorithms for single and double bitstreams in MIMO systems, and the MRC algorithm for combining frequency sub-channels in OFDM. The agreement with measurements in RIMP is typically better than STD of $0.5 \mathrm{~dB}$.

The reverberation chamber has also been used together with the USRP system of National Instruments, a Software Defined Radio (SDR) [14]. Then, it was possible to test also algorithms that are not included yet in commercial devices, such as SVD (Single Value Decomposition) [15]. The results correspond to the theoretical i.i.d. cases in communications system literature.
The introduction of the threshold receiver has made it possible to include both the signal processing algorithm and the number of bitstreams in the characterization of the antennas. The throughput will depend on the antenna performance, the algorithms used, the chosen number of bitstreams, and the environment.
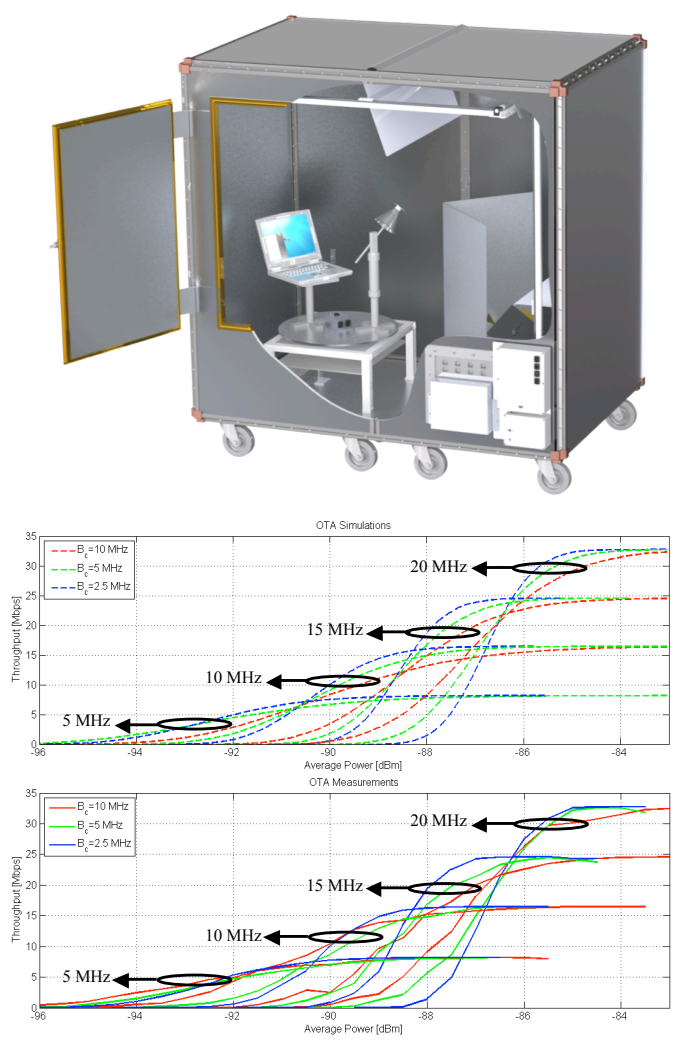

Fig. 4. Drawing of Bluetest's RTS60 reverberation chamber for testing of LTE devices in RIMP. More than 200 reverberation test systems have been sold worldwide till now. The lower two graphs show calculated (upper graph) and measured (lower graph) throughputs of an LTE device [13] for different system bandwidths and coherence bandwidths of the environment. The throughput is the same as a Probability of Detection (PoD).

\section{B. Real-life environments and Random-LOS}

The threshold receiver made it possible to see that the throughput measured in RIMP actually is a Probability of Detection (PoD). This simplified the understanding of the OTA approach so much that it could easily be extended to other types of statistical variations than the fast fading in RIMP. Thereby, real-life environments could be structured into two limiting (or edge) environments, i.e. the RIMP and RandomLOS, that are linked together by a real-life hypothesis: "If a wireless device is tested with good performance in both pureLOS and RIMP environments, it will also perform well in reallife environments and situations, in a statistical sense.’[16][17]

The Random-LOS is also described in the Introduction as an environment with a single dominant wave contributing to the channel. This will most often be a LOS contribution (and that is the reason for the name), but it can also be a major diffracted or scattered contribution. The user terminal will in Random-LOS perceive a slow fading if it is moving. However, the effect of the user statistics is also present for stationary 
users, but then it must be studied over an ensemble of possible user positions and orientations. This characteristic of the slow fading led us to introduce the term Random-LOS for describing it. The Random-LOS is ideally the extreme case of having only one single wave contributing to the received voltage at the antenna port of the user terminal (or base station). The randomness appears because the Angle-of-Arrival (AoA) and polarization are unknown due to the arbitrary orientation and location of at least one side on the link. The user terminal has always a random orientation and location, but sometimes also the base station. The latter appears normally for micro-base stations that are mounted by a user lacking the required skills, i.e. a non-professional person.

A multi-port micro-base station antenna is the first antenna investigated for its performance in both RIMP and RandomLOS [18].

It is clear that both the RIMP and Random-LOS scenarios will be important in $5 \mathrm{G}$, depending on local conditions and the frequency. Therefore, we must ensure that the new devices work for both these extreme cases.

\section{Random-LOS for $4 G$ LTE to cars on the road}

The Random-LOS scenario will also dominate for some $4 \mathrm{G}$ situations, such as for cars on the road. Then, the cars will normally be far away from buildings and other scatterers, and we will often see the base station. Thus, the Random-LOS approach is relevant.

Therefore, we are first developing the Random-LOS test approach for LTE communication to cars. The advantage is that measurement instruments and hardware are commercially available, and in addition we can benefit from all the experience that Chalmers and Bluetest have with modeling and measuring LTE data rate in RIMP, respectively.

The first measurements have been done on a Volvo XC90 in a simplified setup in a semi-anechoic EMC chamber [19] (Figure 5). The results look very promising and shows many of the good features of the Random-LOS concept such as that the probability of detection $(\mathrm{PoD})$ is a much more convenient way of presenting the performance than radiation patterns when randomness is present. The $\mathrm{PoD}$ provides directly a unique quality measure at, e.g., 90\% level (Figure 6).

The Random-LOS setup will be extended with a near-field array in the chamber in order to account for the effect on performance due to the shape of the car, and locations of the MIMO antenna elements at different places on the car. The latter may be needed in order to allow for more than one bitstream in Random-LOS. Some initial studies will be presented in [20].

\section{LIMITATIONS ON NUMBER OF BITSTREAMS AND ANGUlar COVERAGES WITH MASSIVE MIMO}

\section{A. Maximum 2 bitstreams in Random-LOS}

The MRC, ZF and SVD algorithms are very general. Thus, they work equally well in RIMP as in Random-LOS. Still, there are limitations in Random-LOS that are not present in RIMP. For example, it is not possible to form more than two beams (i.e. two eigenchannels) in Random-LOS, and thereby transfer a maximum of two bitstreams. The two eigenchannels corresponds to two orthogonally polarized waves propagating between one transmitting antenna and one receiving antenna.
There is a possibility to get more bitstreams if the antenna on either the transmitting or the receiving side consists of two parts that are separated by several wavelengths. Such large separation will be possible for a multi-user scenario, but normally not for single users.

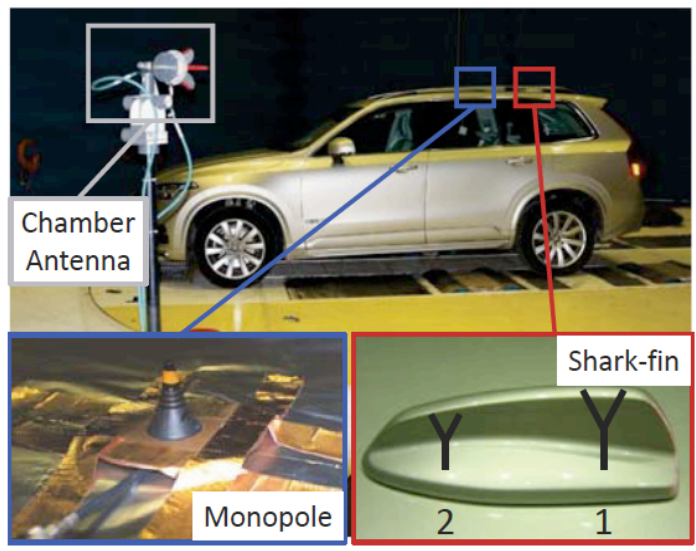

Fig. 5. Initial Random-LOS setup in semi-anechoic EMC chamber for testing LTE communication to cars.
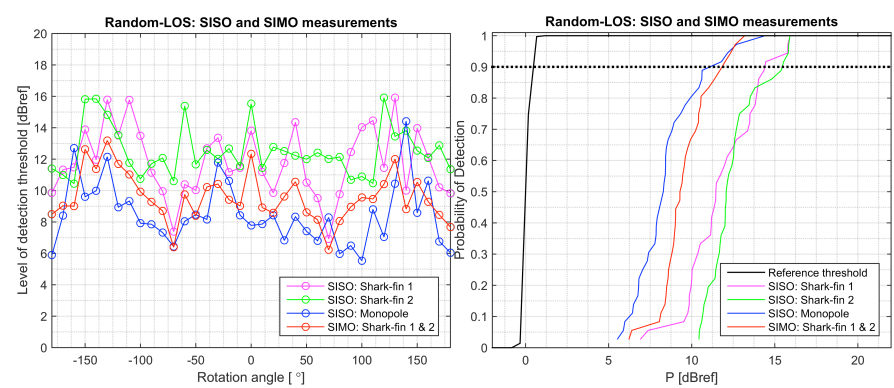

Fig. 6. Initial results of measuring throughput to an LTE devices inside a car with different roofmounted antennas. The upper graphs shows inverse "radiation pattern" type results versus rotation angle, i.e. azimuth angle. They are inverse radiation pattern curves because the lowest points on the curves are the best, i.e. the lower threshold needed to detect the bitstream the better performance. The lower graph shows the same curves when they are presented as PoD curves considering the rotation angle to be random. This is much easier to read and can be used to quantify the difference between the different cases. The presented thresholds and PoDs are in this initial case evaluated for given vertical polarization.

In RIMP there are no such limitations if the environment is rich enough, i.e., if there are enough scatterers and enough power on the transmitting side. The path loss is much larger in RIMP than in Random-LOS.

\section{B. Isotropic coverage with $4.8 \mathrm{dBi}$ directivity}

In $5 \mathrm{G}$ systems there will in many cases be desirable to have omnidirectional or even isotropic coverage. This is of course not possible with planar antenna solutions unless several planar antennas are mounted together to form a cube or similar. The polarization needs also to be adaptable on order to ensure high PoD. This adds a complexity that may not be practical in directive base stations. However, in principle it is enough with two orthogonal polarizations on one side of the link in order to get a high PoD.

Theoretically, it is possible to use the MRC algorithm to linearly combine three orthogonal short dipoles to a beam with $1.8 \mathrm{dBi}$ directivity in any direction and for any polarization. 
The same is possible with three orthogonal magnetic dipoles. By adding them together to a 6-port antenna it is possible to get $4.8 \mathrm{dBi}$ directivity in any direction with MRC. This is also possible by 6 Huygens sources, all being explained in [21], see also Figure 7.

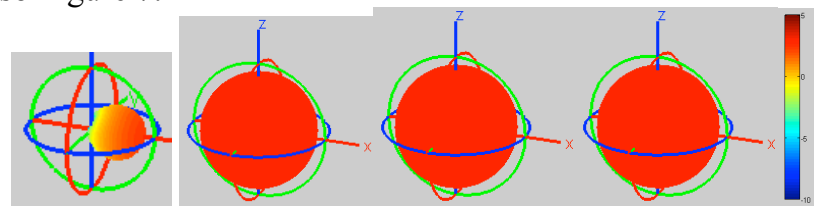

Fig. 7. Radiation pattern of a single Huygens source (left). The three next graphs show the coverage patterns of 6 opposite-orthogonal MRC combined Huygens sources for an incident wave of vertical, horizontal and random polarization. All patterns show a directivity of $4.8 \mathrm{dBi}$. The color scale ranges from -10 to $5 \mathrm{dBi}$.

\section{CONCLUSION}

The paper has given an overview of how we in the Antenna Systems Division at Chalmers prepare for contributing to the developments of $5 \mathrm{G}$ antenna technology. The main focus is on planar steerable millimeter wave antennas. We think there will be needed new waveguide technologies that can be used both for the antenna itself and for integrating it with filters and MMIC modules for the rest of the RF parts and the downconversion. The gap waveguide has already proven to have attractive characteristics for such overall millimeter wave integration and PMC packaging.

Reference [22] gives a historical summary of the developments of the concepts leading to the gap waveguides, starting in 1988 with defining soft and hard surfaces. The developments of the gap waveguides themselves are summarized in [23]. The characterization in RIMP and Random-LOS is conveniently done by the free in-house code ViRMlab [24].

\section{ACKNOWLEDGMENT}

The last years of the work described here have been funded by European Research Council via an advanced investigator grant ERC-2012-ADG_20120216 on gap waveguides, and by Swedish Governmental Agency for Innovation Systems (VINNOVA) within the VINN Excellence Center Chase at Chalmers.

\section{REFERENCES}

[1] T. S. Rappaport, Shu Sun, R. Mayrus, Hang Zhao, Y. Azar, K. Wang, G. N. Wong, J. K. Schulz, M. Samimi, F. Gutierrez, "Millimeter Wave Mobile Communications for 5G Cellular: It Will Work!", IEEE Access, Vol. 1, pp. 335-349, 2013.

[2] P.-S. Kildal, J. Yang, "Some reflector and feed antenna inventions that made a difference", IEEE Africon 2013, Mauritius, 9-12 Sep. 2013.

[3] P.-S. Kildal, "Definition of artificially soft and hard surfaces for electromagnetic waves," Electronics Letters, vol. 24, pp. 168-70, 1988.

[4] P.-S. Kildal, A. A. Kishk, and S. Maci, "Special issue on artificial magnetic conductors, soft/hard surfaces, and other complex surfaces," IEEE Trans. Antennas Propagat., vol. 53, pp. 2-7, jan. 2005.

[5] P. -S. Kildal, E. Alfonso, A. Valero-Nogueira, and E. Rajo-Iglesias, "Local metamaterial-based waveguides in gaps between parallel metal plates," IEEE Antenna Wireless Propag. Lett., vol. 8, pp. 84-87, 2009.

[6] E. Alfonso Alos, A. U. Zaman, and Per-Simon Kildal, "Ka-band Gap Waveguide Coupled-Resonator Filter for Radio Link Diplexer
Application", IEEE Transactions on Components, Packaging and Manufacturing Technology, Vol. 3, No. 5, pp. 870-879, November 2012.

[7] A.U. Zaman, T. Vukusic, M. Alexanderson, P.-S. Kildal, "Gap Waveguide PMC Packaging for Improved Isolation of Circuit Components in High Frequency Microwave Modules," IEEE Transactions on Components, Packaging and Manufacturing Technology, vol. 4, no. 1, pp. 16-25, Jul. 2013.

[8] A. Vosoogh, P.-S. Kildal, "High Efficiency $2 \times 2$ Cavity-Backed Slot Sub-array for $60 \mathrm{GHz}$ Planar Array Antenna Based on Gap Technology," ISAP 2015, Hogart, Australia, Nov. 2015.

[9] P.-S. Kildal, K. Rosengren, J. Byun, J. Lee, "Definition of effective diversity gain and how to measure it in a reverberation chamber", Microwave and Optical Technology Letters, Vol. 34, No 1, pp. 56-59, July 5, 2002.

[10] P.-S. Kildal and K. Rosengren, "Correlation and capacity of MIMO systems and mutual coupling, radiation efficiency and diversity gain of their antennas: Simulations and measurements in reverberation chamber", IEEE Communications Magazine, vol. 42, no. 12, pp. $102-$ 112, Dec. 2004

[11] P.-S. Kildal, C. Orlenius, J. Carlsson, "OTA Testing in Multipath of Antennas and Wireless Devices with MIMO and OFDM", Proceedings of the IEEE, Vol. 100, No. 7, pp. 2145-2157, July 2012.

[12] P.-S. Kildal, A. Hussain, X. Chen, C. Orlenius, A. Skårbratt, J. Åsberg, T. Svensson, T. Eriksson, "Threshold Receiver Model for Throughput of Wireless Devices with MIMO and Frequency Diversity Measured in Reverberation Chamber," IEEE Antennas and Propagation Wireless Letters, Vol. 10, pp. 1201-1204, 2011.

[13] A. Hussain, P-S. Kildal and A. A. Glazunov, "Interpreting the Total Isotropic Sensitivity and Diversity Gain of LTE-enabled wireless devices from Over The Air Throughput Measurements in Reverberation Chambers", IEEE Access, Vol. 3, pp. 131-145, 9 March 2015.

[14] A. Hussain, B. T. Einarsson, P.-S. Kildal, "MIMO OTA Testing of Communication System Using SDRs in Reverberation Chamber", IEEE Antennas and Propagation Magazine, Vol. 57, No. 2, April 2015.

[15] X. Chen, B. T. Einarsson, P.-S. Kildal, "Improved MIMO Throughput with Inverse Power Allocation Study using USRP Measurement in Reverberation Chamber", IEEE Antennas and Wireless Propagation Letters (AWPL), Vol. 13, pp. 1494 - 1496, 2014.

[16] P.-S. Kildal and J. Carlsson, "New approach to OTA testing: RIMP and pure-LOS reference environments amp; a hypothesis," in $E u C A P$ 2013, Apr. 2013, pp. 315-318.

[17] P.-S. Kildal, "Rethinking the Wireless Channel for OTA testing and Network Optimization by Including User Statistics: RIMP, pure-LOS, Throughput and Detection Probability," in ISAP 2013, Nanjing, China, Oct. 2013.

[18] P.-S. Kildal, X. Chen, M. Gustafsson, Z. Shen, "MIMO Characterization on System Level of $5 \mathrm{G}$ Micro Base Stations Subject to Randomness in LOS", IEEE Access, Vol. 22, pp. 1062-1075, Sept. 18, 2014.

[19] M. S. Kildal, J. Kvarnstrand, J. Carlsson, A. A. Glazunov, A. Majidzadeh and P.-S. Kildal, "Initial Measured OTA Throughput of 4G LTE Communication to Cars with Roof-Mounted Antennas in 2D Random-LOS”, ISAP 2015, Hobart, Australia, Nov. 2015.

[20] A. A. Glazunov, P.-S. Kildal, and M. S. Kildal, "Devising a Horizontal Chamber Array for Automotive OTA Tests in Random Line-Of-Sight", ISAP 2015, Hogart, Australia, Nov. 2015.

[21] Per-Simon Kildal, Xiaoming Chen, "Fundamental Limitations on Small Multi-Beam Antennas for MIMO Systems", in EuCAP 2014, The Hague, The Netherlands, 6-11 April 2014.

[22] P.-S. Kildal, "Metasurfing for industrial applications since 1988", Slide presentation published in web based FERMAT Journal edited by R. Mittra, Aug. 2015.

[23] A. Uz Zaman, P.-S. Kildal, "Gap waveguides for mmWave antenna systems and electronic packaging", chapter to appear in Handbook of Antenna Technologies, Springer 2016.

[24] U. Carlberg, J. Carlsson, A. Hussain, and P. S. Kildal, "Ray based multipath simulation tool for studying convergence and estimating ergodic capacity and diversity gain for antennas with given far-field functions," in 20th Internat. Conf. Applied Electromagn. and Comm. (ICECom), 20-23 Sept. 2010, Dubrovnik, Kroatia, 2010. 
